\title{
PRODUCTOS Y ORGANIZACIÓN TÉCNICA DEL TRABAJO EN AZAPA DURANTE EL SIGLO XVIII: PONIENDO CHACRA DE AJÍ, COGIENDO ACEITUNAS
}

\author{
PRODUCTS AND TECHNICAL LABOR ORGANIZATION IN \\ AZAPA VALLEY DURING AD XVIII CENTURY: PLANTING CHILI \\ PEPPER FIELD, PICKING OLIVES
}

Francisco Henríquez*

\begin{abstract}
Se analiza la producción y las técnicas agrícolas del valle de Azapa durante el siglo XVIII en el contexto de la economía regional Centro Sur Andina. Los cultivos del ají y la aceituna nos permitirán apreciar cómo la actividad productiva del valle se vio condicionada por las transformaciones macroeconómicas del período. Al observar estos dos cultivos, no se desconoce la existencia de otros cultivos comerciales en esta zona, pero se privilegió el estudio del ají y la aceituna por ser los principales en términos mercantiles y por ser los más relevantes en la organización de la agricultura azapeña en aquella época.

Palabras claves: Ají, aceite, Azapa, organización técnica del trabajo.
\end{abstract}

The production and the agricultural techniques of Azapa, during eighteen century will be analyzed in the context of the regional economy. The cultures of the red pepper and the olive, will allow us to appreciate how the productive activity of the valley was conditioned by the macroeconomic transformations of the period. In observing these two cultures, we will not fail to recognize the existence of other commercial plants in the zone. However, they have the privilege of being studied due to they were the main ones in mercantile terms and the most prominent in the organization of agriculture in the valley in that time.

Key words: Chili pepper, oil, Azapa, technical organization of the work.

Andaluces de Jaén, / aceituneros altivos, / decidme en el alma: ¿Quién, / quién levantó los olivos? / No los levantó la nada, / ni el dinero, ni el señor, / sino la tierra callada, / el trabajo y el sudor. /Unidos al agua pura / y a los planetas unidos, / los tres dieron la hermosura / de los troncos retorcidos. /Levántate, olivo cano, /dijeron al pie del viento. /Y el olivo alzó una mano / poderosa de cimiento (Hernández 2000: 53).

Miguel Hernández, en su poema "Aceituneros", al recorrer los campos de Jaén y observar las añosas plantaciones de olivares, se preguntó cómo fue posible que tan grande espectáculo a la vista del viajero se desplegara. Y, con la particular sensibilidad del vate, logró ver más allá de los macizos e imponentes troncos de los olivos, y encontrar al hombre, a los cientos y miles de hombres, que hicieron que la tierra fuera pródiga en uno de los manjares más apetecidos por la cultura occidental desde hace más de tres mil años. En este marco, se tratará de dar cuenta de cómo, a fines del período colonial, los individuos, esto es, los campesinos y hacendados que convivían en las desérticas tierras de Arica, lucharon contra los elementos para que fructificara el valle de Azapa; y cómo lo hicieron para poder subsistir y progresar en un mundo, como el de fines de la colonia, en el cual poco a poco la sociedad mercantil estaba empezando a teñir las relaciones sociales de producción. Dentro de este contexto, el de la mercancía y la economía mercantil, se tratará de buscar una explicación al cambio de producción desde el ají hacia el olivo, detallando la particularidad del trabajo de cada cultivo, lo que le da sentido y materialidad a toda discusión sobre estos productos y, en general, a la manera en que reproducían su existencia los antiguos habitantes de Arica durante el siglo XVIII.

Para lograr esto, se hará un análisis necesariamente abstracto del proceso de trabajo, ya que no se tomarán en cuenta las condiciones sociales en que se realizaba, lo que ya fue analizado ${ }^{1}$. Luego, se efectuará una exposición de las características generales de los procesos técnicos de trabajo y del

* Departamento de Arqueología y Museología. Universidad de Tarapacá, casilla 6D, Arica, Chile. frahenpal@ yahoo.com 
devenir que tuvieron los productos en el período que se estudia. Para ello se utilizará como guía la metodología empleada por Garavaglia (1983) para el análisis de la producción de la yerba mate en Paraguay durante el período colonial. En este trabajo se tomaron en cuenta para estudiar las relaciones de producción, en primer lugar, las características de ese bien, los principios generales de la tarea de cooperación y los mecanismos técnicos que se utilizaban en su producción, es decir, la organización técnica del trabajo (Garavaglia 1983: 243) $)^{2}$; y en segundo lugar, las indicaciones de Assadourian respecto a los circuitos económicos que se estaban articulando en América Latina a raíz de la producción de plata y la implementación de grandes centros administrativos coloniales (Assadourian 1982). Sobre la base de esto, se estudiará la producción de dos bienes que fueron los ejes de la producción de Azapa y de la zona durante el siglo XVIII, principalmente por su carácter mercantil: el ají y el olivo (Hidalgo 1993).

Por la documentación a la que se ha tenido acceso, de carácter judicial y notarial, se ha podido determinar que hasta mediados del siglo XVII, si bien la producción de ají y olivo se realizaba en el valle, la más importante era la de vinos, la cual dejó de tener relevancia a favor de aquellos productos a partir de mediados del siglo XVII. Esto no implicó que desapareciera del valle, ya que aún hay rastros de viñas en el siglo XIX, sino que no era lo que se llevaba la mayor cantidad de inversiones ni generaba la mayor proporción de ganancias. Así el viajero Frezier, quien visitó las costas sudamericanas a comienzos de siglo, afirmaba que desde San Miguel sólo se producía ají (Frezier 1902:152). En cambio, un siglo más tarde, el diligente alcalde Ponciano Jofré de Osorio (ANA 1804), en respuesta a la solicitud de un informe que describiera la zona, sostuvo que "[...] el renglon de mas consideracion de estos [productos] es el de la aceytuna"... ${ }^{3}$. Reiteramos, esto no significa que mientras predominaba uno, no se cultivara el otro, tampoco que no se produjera ningún otro producto en el valle. Es más, por la condición de valle dulce -con agua sin muy alta concentración de sales-, había una gran diversidad de plantas cultivándose; sin embargo, por ser estas dos las principales, y por tener mayor información sobre ellas, es que se analizará cómo se produjeron. No obstante lo anterior, se darán a conocer las diversas plantas que se utilizaron agrícolamente en el valle a fines del período colonial.

\section{Geografía de Azapa: Descripción de un Valle Dulce}

El valle de Azapa se ubica al este de la ciudad de Arica, y poseía una extensión de 30 kilómetros en la zona donde se concentraban las haciendas, es decir, las de "Las Chimbas" y "Saucachi-Las Riveras" (Keller 1946:142) (Figura 1). Actualmente, en el valle hay 2.144 ha arables (Instituto Geográfico Militar 1985: 61). De esa área, hay 1.050 ha que recientemente se pudieron utilizar en la agricultura debido principalmente al aprovechamiento de aguas del río Desaguadero a través del canal Lauca (Errázuriz et al. 1987:226). El tramo específico del comienzo del valle, que según Keller abarcaba 647 ha (Keller 1946:142), se caracteriza, como sostuvo Rivera (1983:14), por “un clima propicio y un adecuado espacio de tierras fértiles para poder cultivar frutos tropicales y templados. No obstante, éste presentó dos inconvenientes en la época colonial: de una parte, no tenía agua permanente y suficiente para cultivar todas las zonas apropiadas, y de otra, por las características de ciénaga que tenían algunos lugares (v. gr., Ocurica y Las Chimbas) estaba expuesto a malarias". Los suelos son también alcalinos y con poca materia orgánica (Corporación de Fomento de la Producción [CORFO] 1950:333), lo que hace necesario abonarlos sistemáticamente para que fructifiquen. $\mathrm{La}$ hidrografía del valle se caracteriza por la existencia de una cuenca exorreica (Errázuriz et al. 1987:104), con un lecho de río hasta la costa, que en gran parte de su curso hacia la desembocadura, por las características desérticas de la zona, no muestra aguas superficiales. Esta falta de una corriente de agua periódica se contrarrestaba con el uso de vertientes, que se denominan puquios. Sin embargo, estas vertientes son insuficientes para las necesidades agrícolas del valle. El agua, no obstante, se puede describir como dulce, es decir, sin una concentración muy alta de sales como en Lluta. El clima, según la clasificación hecha por CORFO, es árido subtropical, caracterizado por una progresiva aridez. La carencia de precipitaciones es paliada por la abundante niebla o camanchaca (Rostworowski 1981:21, 34; Henríquez 1998:37). 


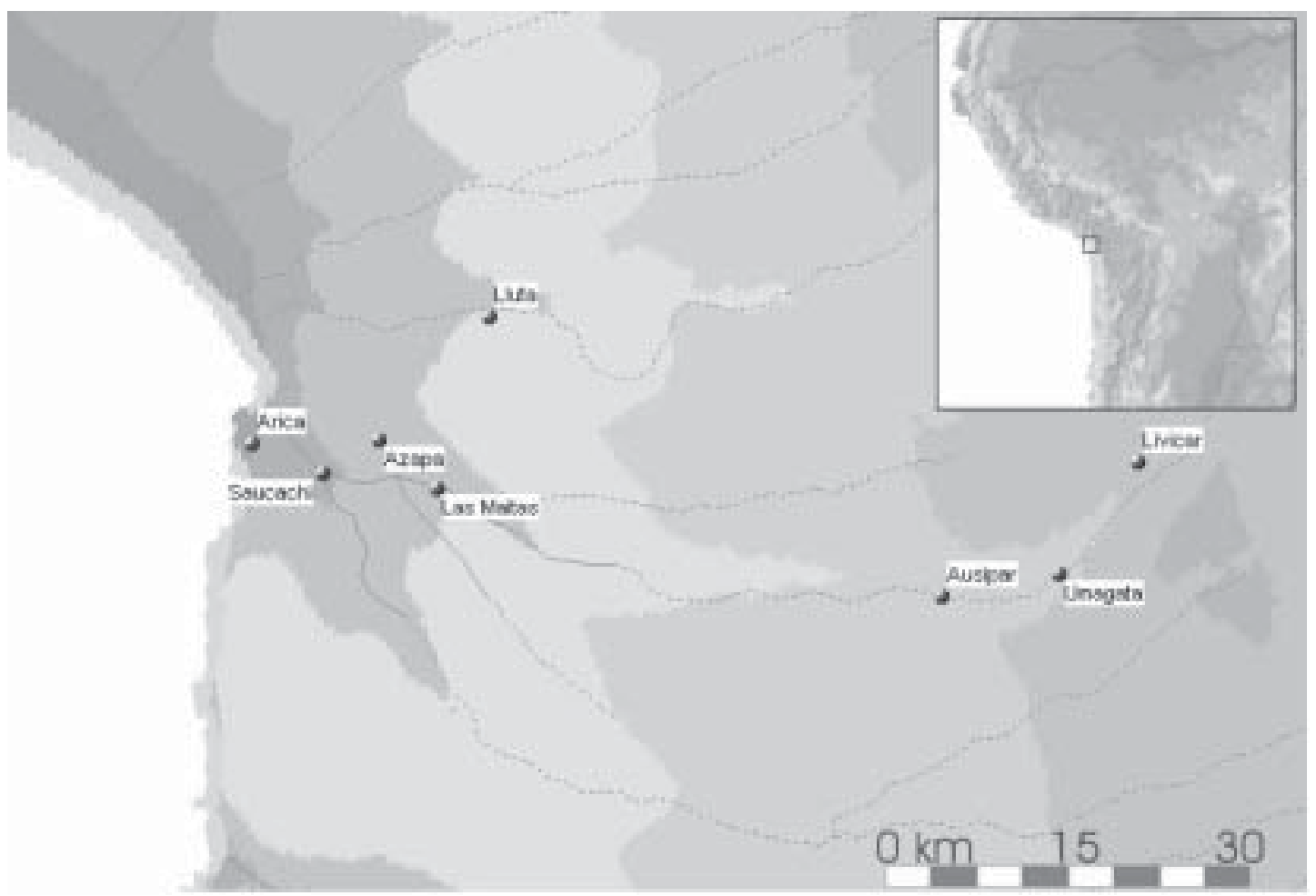

Figura 1. Mapa de la Zona de Arica y Azapa.

Map of the Arica zone and Azapa.

Las características del valle responden a lo que Álvarez (1992:149-153) calificó como valles dulces, en oposición a los valles salados. Estos valles tienen la característica de poseer tierra fértil, un clima templado y agua escasa pero dulce, lo que da lugar a una gama muy amplia de cultivos, pero a una escala limitada. Esta amplia gama de cultivos atrajo desde comienzos del período colonial a los españoles a asentarse en él, procurando cultivar plantas de una importante significación mercantil (Hidalgo 1993). Es así que, como ya se indicó, se diera preferencia a cultivos como la vid, el ají y el olivo para poder obtener de ellos ganancias en el mercado colonial.

\section{El Ají y el Mercado Indígena}

A pesar de que algunos autores, como Romano (1992), cuestionan la relevancia que tuvieron, en los circuitos económicos regionales, los productos de consumo mayoritario en las clases bajas, otros, como Assadourian (1982:164), han seguido la pista de este tipo de productos en los circuitos económicos regionales. En nuestra investigación hemos encontrado evidencia coincidente con testimonios como el de Frezier, quien sostenía que el ají era un producto muy apetecido en la zona de la Puna, pero, debido a que no se podía producir allí, los valles costeros debían proveerlo (Frezier 1902:153). La mediación comercial que se produjo en el período colonial para poder obtenerlo, cortando en cierta medida la complementariedad vertical existente en el mundo andino (Murra 1975), hizo que la producción de este bien -convertido en mercancía- se volviera muy atrayente para los colonizadores, los cuales se sintieron estimulados a plantar ají en las tierras que ocuparon en las zonas costeras.

El ají es una planta que pertenece a la familia de las solanáceas, originaria de América, y al género Capsicum que posee además del ají muchas especies, que van desde el pimiento dulce al ají picante. Es muy utilizado en todas sus variantes como alimento. En la época colonial se consumía fresco y seco. Esta última modalidad permite que se conserve por mucho tiempo y sea liviano, lo que hacía posible y rentable su transporte a lejanas regiones, estimulando su comercialización. 
Según el relato de Frezier, cuando pasó por Arica en 1713, a una legua de esa ciudad, en el valle de Sapa se cultivaba ají “"[...] con lo que está plantado todo el resto del valle [desde la aldea de San Miguel hacia arriba ${ }^{5}$ ] i sembrada de alquerias [parcelas], únicamente ocupadas por esta legumbre"... (Frezier 1902:152). Debido a esto, se vendían más de 80.000 pesos anuales, produciéndose en conjunto con los valles de Tacna, Sama y Locumba un valor de 600.000 pesos anuales. Según él, era el principal producto del valle en la primera mitad del siglo XVIII.

Afirma que el secreto de esta importante producción fue la utilización de guano, que hacía rendir las semillas de cuatrocientos a quinientos por uno. Y con el objeto de mostrar cómo se debía utilizar para no malograr la producción, describió de qué manera se producía el ají.

Habiendo ejercido el guano i en estado de trasplantado se colocan las plantas serpenteando a fin de que la misma disposición de los surcos que llevaban el agua para regarlos, la conduzca suavemente al pié de las plantas, entonces se echa al pié de cada ají un puñado de guano, lo que cabe en el hueco de la mano. Cuando la flor está formada, se le hecha un buen puñado, teniendo siempre el cuidado de regar porque no llueve jamas en ese pais i las sales que contiene el guano no estando mojado quemarian las plantas, como lo ha hecho notar la experiencia. Por esta razon se echa en diferentes ocasiones con cierto tino, que el uso ha hecho conocer en la diferencia de cosecha (Frezier 1902:153-154).

De este texto se desprende que la producción de ají en el valle de Azapa se realizaba de la siguiente manera:

1. Se comienza con los almácigos, correspondiente a la primera fase de desarrollo de la planta. Hasta que alcanzaba el "estado de trasplantado".

2. Se prepara la tierra con guano o, en términos de Frezier, "se ejerce el guano".

3. Se trasplantan las matas en esta tierra que está dispuesta en forma de "caracol"6, que es un modo de cultivar propio de esta zona, cuya virtud principal es aprovechar al máximo el agua $\mathrm{y}$, como sostiene el viajero, es también condu- cir el agua "suavemente al pie de las plantas", sin producir mucha erosión en el suelo.

4. Se deben regar las plantas, por cuanto no hay lluvias, lo que requería mantener en buen estado las acequias comunales. La limpieza de las acequias comunales ${ }^{7}$ se menciona permanentemente en la documentación consultada, cuando se refiere a las actividades realizadas normalmente en una hacienda.

5. Se le administra periódicamente guano para suplir las carencias de la tierra del valle ${ }^{8}$, en una cantidad de "un puñado, lo que cabe en una mano”. Destacándose que esto se realiza "cuando la flor está formada".

6. Y por último, la cosecha, realizada en mayo, dependía del agua disponible y de la cantidad de guano que se utilizaba en el cultivo de la planta. Lo primero queda en evidencia con la declaración de un arrendador, el cual afirmaba que

sabe y le consta por haberlo visto que Don Balthasar Peres de los Rios aviendole escaseado el agua por la seca general que padecio todo el valle de Asapa y no siendo vastante lo que corria para llegar a su hacienda por el año de setecientos veinte y seis puso chacra de Aji en un pedaso de tierras que le dio este testigo en su hacienda de que coxio cosecha no muy abundante por haberse perdido la dicha chacara 9 .

La fecha de la cosecha queda claramente explicitada en la declaración de Juan Jiménes de Valverde a fines de la década de 1720, quien sostenía "[...] que vio la dicha chacara [de ají] y que fue competente que sabe cogio cosecha de mayo la chacara"... ${ }^{10}$. No se conoce el número exacto de trabajadores que se necesitaban para producir una determinada cantidad de ají, aunque se sabe que una "quantiosa chacara de Aji"11 se benefició "con ocho o diez negros"12. Sin embargo, se puede sostener que por las características mismas del proceso, se ocupaba mayor cantidad de trabajadores en las labores de trasplantado, preparación de la tierra y principalmente de cosecha del fruto, y una porción menor en las actividades de cuidados durante el proceso de desarrollo de la planta. También, por las condiciones mismas del mercado colonial, la mayor parte de las unidades productivas no necesitaban disponer de jornaleros para trans- 
portar el producto, ya que los comerciantes, al parecer, iban a comprar al mismo valle ${ }^{13}$, aun cuando existían quienes eran utilizados para el transporte de guano a las haciendas.

Estos procesos técnicos de trabajo respondían a un mercado determinado y a una realidad regional específica. Gracias al monto en dinero estimado por Frezier para Azapa, es posible calcular cuánto se producía en el valle y en la zona a comienzos del siglo XVIII, ya que hay documentos que poseen el valor de este producto. Por los datos entregados por Ureta y Peralta (1962 [1792]) y Ponciano Jofré Osorio (ANA 1804), se puede acceder a la información para fines del mismo siglo. Como se sostuvo al comienzo del apartado, el mercado que tenía el ají era mayoritariamente el de las clases bajas, aun cuando era un producto consumido por toda la sociedad. Y, según lo señalado por Frezier, el mercado geográfico específico de la producción azapeña era la puna (Frezier 1902:153). Por ello, su precio debió estar muy ligado a la suerte de las capas bajas de la sociedad que vivían en esa zona. Frezier sostiene que Azapa producía 80.000 pesos anuales ${ }^{14}$, y que la zona en total producía un valor de 600.000 pesos en el mismo período. Se sabe que el valor aproximado para el ají era de 4 y 5 pesos por arroba hacia $1730^{15}$ y que en 1760 se vendía a 4 pesos 2 reales $^{16}$. Es decir, se puede considerar que 4,5 pesos por arroba es un precio medio adecuado del ají para la primera mitad del siglo XVIII. Entonces, en la zona se producían aproximadamente $133.333,33$ arrobas de ají (aproximadamente 1.533.332,95 $\mathrm{kilos}^{17}$ ), y en Azapa 17.777,77 arrobas (204.444,44 kg aproximadamente), equivalente a un $13,33 \%$ de la producción del Corregimiento de Arica (Tabla 1).
Por otra parte, a fines de la colonia (siglo XIX) se cosechaban 1.000 arrobas de ají, según la información entregada por Ponciano Jofré Osorio (ANA 1804) ${ }^{18}$. Teniendo en cuenta las cantidades dadas por Ureta y Peralta (1962 [1792]), de 80.000 arrobas al año en el Partido de Arica ${ }^{19}$, se puede establecer que producía un $1,25 \%$ de lo que se cosechaba en la zona. Además, utilizando la misma información dada por Ureta y Peralta (1962 [1792]), también se puede afirmar que el precio del ají estaba entre 1,5 y 3 pesos, dependiendo de la calidad del mismo (Tabla 2). Si se comparan las cifras que se poseen de los dos períodos, se puede afirmar que hubo una disminución absoluta de la cantidad producida de ají en el valle. Esta variación fue del orden del 87,14\% en Azapa y del $39,99 \%$ en la zona. Esta disminución absoluta en el valle significó también un deterioro en la importancia relativa de la cantidad con la que contribuía a la producción regional, ya que pasó de tener el $13,33 \%$ de la producción regional a sólo el 1,25\%.

Es altamente probable que esta disminución haya respondido a las condicionantes que impuso el mercado a los productos americanos durante este período a través de los precios. Tandeter y Wachtel, a la luz de la construcción de series de precios en el mercado de Potosí, concluyen que en el siglo XVIII “[...] casi todos [los productos peruanos] se caracterizan, a pesar de rasgos originales propios de cada caso, por una tendencia general al estancamiento, incluso a la baja, en particular durante la segunda mitad del siglo XVIII" (Tandeter y Wachtel 1983: 41). Esta baja, que se aprecia en las estimaciones que grosso modo se pueden hacer acerca del precio en Azapa, que bajó de la primera a la segunda mitad del siglo XVIII, debió frenar a

Tabla 1. Producción de ají y aceite (en arrobas) y su situación regional. Regional chilli pepper and oil production (measured in arrobas).

\begin{tabular}{lcccccc}
\hline & & Ají (arrobas) & \multicolumn{3}{c}{ Aceite (arrobas) } \\
\hline Años & Azapa & Región & \% Región & Azapa & Región & \% Región \\
\hline 1713 & $7.777,77$ & $133.333,3$ & 13,33 & - & - & - \\
$1775-1804$ & 1.000 & 80.000 & 1,25 & 7.000 a 8.000 & 40.000 & 17,5 a 20 \\
\hline
\end{tabular}

Fuentes: Frezier (1902); Ureta y Peralta (1965); ANA leg. 4 Informe de Ponciano Cofre Osorio en Arica, Arica 1804, fjs. 41 r.-42 v. 
Tabla 2. Producción del Partido de Arica en 1790.

Production of the Partido of Arica in 1790.

\begin{tabular}{lccc}
\hline Frutos & Peso (en arrobas) & Precios & Valor total (en pesos) \\
\hline Algodón & 80.000 & á 16 reales & 160.000 \\
Vino & 90.000 & á 10 reales & 112.500 \\
Axí de carga & $400.000[40.000]$ & á 24 reales & 120.000 \\
Id. regalado & $400.000[40.000]$ & á 12 reales & 60.000 \\
Aceyte & $400.000[40.000]$ & á 24 reales & 120.000 \\
Maíz & 120.000 & á 2 reales & 30.000 \\
\hline Totales & 410.000 & & 602.500 \\
\hline
\end{tabular}

Fuente: (Ureta y Peralta 1965: fol. 132).

los productores a ofertar ese producto, lo cual redundó en una caída de la cantidad que se vendía.

En el caso específico del ají, la causa de esta baja de la cantidad vendida hay que buscarla en el empeoramiento de los niveles de vida de los indígenas (Tandeter 1995:22) y en general de las clases bajas, ya sean mestizas o castas (Tandeter 1995:20). Ello se debió a procesos de disminución de tierras comunales, por el lado de los indígenas, y de apropiación de los minifundios, por parte de los demás (Tandeter 1995) o empeoramiento en general de las condiciones en que se desarrollaban las diversas tareas productivas para los pequeños y medianos propietarios y para los asalariados (Fisher 1977). Además, se deben agregar los progresivos impuestos y demás cargas, tales como los repartimientos, que a través de todo el siglo XVIII impuso la Corona española en el denominado proceso de "Reconquista" de sus colonias de ultramar que se convirtieron en pesadas cargas para la población (O'Phelan 1985). Además de ello hay que agregar un mejoramiento del nivel de vida de las clases acomodadas que estimularon la producción de bienes para su consumo, como el que pasamos a estudiar ahora, el olivo.

\section{El Olivo y el Consumo con Discriminación Social}

Hasta el día de hoy el valle de Azapa es famoso por sus aceitunas. Su producción se remonta por lo menos a la segunda mitad del siglo XVI (Hidalgo 1993:33). Pero no alcanzó su apogeo como producto principal de vinculación mercantil del valle hasta el siglo XVIII. La razón para ello fue que, en los siglos anteriores, las variables condiciones del mercado colonial y las políticas estatales ${ }^{20}$ se dieron más favorables a la producción de vides y de ají sucesivamente.

En un primer momento Azapa suplió la creciente demanda de vino en los mercados regionales, pero fue gradualmente desplazada por los valles de Moquegua (Glave 1983) y Arequipa (Takahashi 1986:121), donde era más económica su producción, en cuanto su extensión permitía tener un mayor nivel de producción que Azapa, con la consecuente disminución de los costos, es decir, con economías de escala.

En un segundo momento, a mediados del siglo XVII (Assadourian 1982:164) la demanda por ají debió aumentar al mismo ritmo en que empezaba a recuperarse la población indígena y a aumentar la población mestiza (Tandeter 1995:8). Pero debió llegar un momento, a mediados del siglo XVIII, en que la presión demográfica, unida a las exacciones que estaban siendo objeto los comuneros y los demás estratos bajos de la población, haya hecho bajar los precios del ajî21. Ello debió significar un estímulo a la variación de la producción, en este caso del aceite, que ya se producía en el valle desde hacía tiempo. Por ello, para conocer qué y cómo se producía en Azapa durante la segunda mitad del siglo XVIII, es importante estudiar el olivo.

El olivo es un árbol muy longevo, originario de la zona del Mediterráneo, se propagó a México, Perú y Chile en el siglo XVI ${ }^{22}$. Las aceitunas producen entre un $10 \%$ a un $12 \%$ de su peso en aceite cuando se procesa para ese fin (Gómez de Fuencarral 1883). Al igual que el ají, luego de ser pro- 
cesado, el fruto se puede conservar por largo tiempo, lo que favorece su transporte a grandes distancias. Lamentablemente no se dispone de un documento que relate cómo se cultivaba este producto en el siglo XVIII. Esta ausencia documental se suple con la revisión de un texto español de fines del siglo pasado. Para estudiar las especificidades de su producción en Azapa, se revisan dos rendiciones de cuentas como, además de otros documentos, dividiendo la descripción en trabajo cultural -el relacionado con el cultivo- y elaboración del fruto.

La primera rendición de cuentas es la que entregó Manuel Calderón respecto a la hacienda de su padre, José Calderón, en un pleito que llevó contra Joaquina Rivera, su madrastra. La segunda es un testimonio de Ramón Pimentel, que fue el encargado de administrar la hacienda Arias debido a un embargo que sufrió el dueño de ella, Ventura Iglesias, por parte de Manuel Albarracín. En relación con las rendiciones de cuentas, la Tabla 3 muestra esquemáticamente las diversas actividades productivas que se realizaron en la hacienda de José Calderón, entregando una breve descripción de la actividad y los días/hombre que se contrató para realizarlas. Es relevante debido a que en esta hacienda sólo se cultivaba olivos a gran escala con fines comerciales, por lo tanto se mencionan las labores que estaban más directamente relacionadas con esa actividad. En su exposición se ha seguido el orden indicado en el documento, principalmente porque la secuencia presta una valiosa información sobre la manera en que se realizaban los procesos productivos en Azapa.

\section{Labores culturales}

Según Gómez de Fuencarral, había que labrar la tierra una o dos veces al año, después de la temporada de heladas. Se debía podar a fines del invierno y desyemar a fines del verano. También se necesitaba abonar periódicamente. Y en zonas como Azapa, donde no hay lluvias, era importante el riego, aunque no en cantidades excesivas. El riego es indispensable en invierno, útil en otoño y perjudicial en verano. Por último, los frutos se recolectaban en otoño e invierno, vareando los árboles, es decir, golpeándolos -lo que no se recomendaba por posibles daños al fruto- o cogiendo las aceitunas una por una en verano (Gómez de Fuencarral 1883). Con respecto a lo que pasaba en Azapa, y tomando en cuenta lo anterior y lo entregado en la Tabla 3, podríamos sistematizar de la manera siguiente el proceso productivo:

1. Se contrataba gente para regar. El documento más tardío sostiene explícitamente que se regaba el olivar mediante empozamientos. Este es un modo de riego distinto al caracol y consiste en excavar una taza no muy profunda alrededor del árbol para inundarlo ${ }^{23}$. Esta es una práctica hispana que tiene la desventaja de desaprovechar el agua. Las haciendas, según las fuentes, se regaban con distintos tipos de aguas: mita, días, estancadas y avenidas del río ${ }^{24}$. Las mitas son turnos periódicos acordados por los comuneros en los cuales se tiene derecho a utilizar el agua de una fuente común, no importando su caudal, los cuales pueden ser de unas pocas horas, medio día o un día completo (Burga 1976:41). Los días, como su nombre lo indica, son

Tabla 3. Actividades realizadas por los trabajadores en la hacienda de Calderón en el período 1781-1782. Activities carried out by workers in the Calderon's Hacienda, 1781-1782 period.

\begin{tabular}{lr}
\hline Actividades & Días/hombre \\
\hline Saca y limpia primera de la acequia principal & 9 \\
Roza del olivar & 84 \\
Poda del olivar & 29 \\
Sacar de dentro del olivar el chiñe y la ramazón & 16 \\
Saca de la acequia del olivar de arriba la principal & 14 \\
Ayudar a limpiar la acequia del manantial & 7 \\
Limpia de la acequia regadera del todo el año & 57 \\
Riego & 32 \\
Recojo de aceituna de 1781 & 133 \\
Cargar sal & 11 \\
Moler sal & 16 \\
Cargar aceituna al molino & 7 \\
Moler aceituna & 18 \\
Peón de leña para el molino & 6 \\
Mayordomo que asistió a la molienda & 6 \\
Mayordomo para recoger la aceituna & 16 \\
Recojo de la aceituna & 60 \\
Cargar sal & 8 \\
Moler sal para la aceituna & 3 \\
Moler aceituna & 9 \\
Cargar la aceituna al molino & 8 \\
Mayordomo que asistió al molino & 3 \\
Mayordomo en el recojo de la aceituna & 3 \\
& 4 \\
&
\end{tabular}

Fuente: AJA, leg. 22, pza. José Calderón. División de bienes en Azapa, Azapa 1781-1784, fjs. 25 r.-25 v. 
un tipo de mita que se compone de días enteros de disposición de agua para el que la adquiere. Las estancadas es una cantidad de agua medida en volumen, de un estanque, a la cual tiene derecho un comunero, no importando cuánto se demore en llenarse el estanque. Y las aguas de avenidas del río son las que trae el lecho del río en los años que llega al valle, los que son eventuales ${ }^{25}$.

En ambos documentos hay un interés especial por parte de los interesados de las rentas de que el agua riegue el olivar o los cultivos mercantilmente más apreciados. Esto provocó un conflicto con los que dependían directamente de las haciendas, es decir, los que las administraban, debido a que éstos pretendían sistemáticamente mantener cultivos mixtos, tal vez con el fin de sortear de mejor manera los caprichos del clima, determinantes para los agricultores de épocas preindustriales (Wrigley 1994:77). En la década de 1780, por ejemplo, la beneficiaria de la hacienda de José Calderón, que estaba en disputa, reclamaba que las aguas compradas por el encargado de administrarla, su hijastro Manuel Calderón, no

han sido nesesarias para el riego de los olivares de la Hacienda por tener sufisiente con la propia para el riego de estos: Hantes si perjudicando esta finca ha suspendido el agua absolutamente de ella invirtiendola en un cresido alfalfar que tiene en dicha hacienda fuera de los olivares ${ }^{26}$.

Y en la hacienda Arias, debido a que se encontraba en malas condiciones, un grupo de peri$\operatorname{tos}^{27}$ a los que se les consultó sobre lo que se debía hacer para mejorarla dieron como primera medida que se ha

de dar la hasienda y el caracol de agua gratis por el termino de un año sin gravamen ni pension alguna para que labre precisamente las tierras que ocupa el olivar y algodonal y no otras [el subrayado es nuestro], con el fin de ver si los olivos con la humedad y cultivo reviven, y lo mismo los algodonales ${ }^{28}$.

Dentro de esta misma hacienda, el que pidió embargarla, Manuel Albarracín, haciendo una evaluación de la gestión del que quedó a cargo de la hacienda, Ramón Pimentel, sostuvo que si hubiera aplicado todas las aguas a aquellos dos unicos objetos de su contrato [algodonales y olivares], bien [ilegible] que son los que hacen la base de la hacienda y no las hubiera substraido para cultivar otras chacras fuera de ello contraviniendo el instrumento de arriendo: hubiera usufructuado un numero quintiplicado de arrobas en ambas especies, $y$ la Hacienda estuviera hoy en un pie mas ventajoso, y apreciable ${ }^{29}$.

Todo esto significa que para los que sólo percibían rentas, el escaso elemento era preferente para este tipo de cultivos, en menoscabo de la variedad de producción, lo cual debió perjudicar a los que dependían directamente de las haciendas ya que a largo plazo los monocultivos en la época colonial hacían muy vulnerables a las personas y a los ne$\operatorname{gocios}^{30}$.

Con respecto a las actividades relacionadas directamente con el agua, en ambos documentos hay indicaciones precisas de contrato de regadores, además de peones para trabajos privados y colectivos en las obras de riego del valle. En este sentido, se hace mención a los encargados de regar. Es más, en la hacienda Arias se contrató a un regador exclusivo por el lapso de tiempo del que se tiene información ${ }^{31}$, aparte de todos los demás regadores que se contrataron para tareas de un día. Por otro lado, se hace clara mención a las actividades colectivas en los dos documentos. En la hacienda Arias se contrató gente para realizar trabajos en la Media Luna ${ }^{32}$, para "sacar la sequia" 33 y para "cargar champas para el estanque de la Media Luna" 34 . Y en el caso de la hacienda de José Calderón, se sostiene claramente que se contrató a "siete peones" "para aiudar a limpiar la sequia del manantial por ser obligasion [el subrayado es nuestro]"35. Esta última cita deja en claro el carácter obligatorio que tenían para los hacendados las actividades vinculadas con el interés común, específicamente las que se refieren al uso y mantención de ciertas obras públicas.

2. Aun cuando el documento más temprano no da información sobre el abono ni el laboreo de la tierra, sostiene que había un camayo ${ }^{36}$ permanente en la hacienda, presumiblemente para realizar estas labores, ya que la paga que percibió nos hace presumir que estuviera a cargo de los demás trabajadores $^{37}$. Esta afirmación se basa en que, en la 
segunda rendición de cuentas, sí se entregan datos sobre el "guaneo", "laboreo", "arado" y "lampa" (utilizar esa herramienta), algunas de estas actividades explícitamente relacionadas con el olivar, tales como "rozar el olivar", "labrar el olivarcito" y "arar el olivarcito"38. Dentro de estas tareas, en ambos casos encontramos los trabajos de "roza", que consistían en quemar las ramas secas producto de la poda, como primera actividad mencionada. Y en la rendición de cuentas de la hacienda Arias, se menciona sistemáticamente el "basureo" $(\text { limpieza) })^{39}$.

3. En cuanto a la poda y desyemación de los olivos, en el primer documento, referido a la hacienda de José Calderón, se menciona la actividad de "poda" y de la "saca del chiñe y la armazón"40. El segundo da cuenta de las tareas de "chapodar" y "arreglar el olivar" 41 ; labores que se realizaban una vez al año en medio de otras actividades que se practicaban durante todo el año ${ }^{42}$.

4. Y en el ámbito de las actividades de cosecha de la aceituna -actualmente denominada "raima" en el valle-, se hacen menciones explícitas en los dos documentos. El documento de los años 1780 describe actividades de recojo de la aceituna, contratándose un mayordomo para su supervisión en los dos años en que se realizaron cosechas. En el documento de los años 1810 se da cuenta de las dos maneras de cosecha: vareándola y cogiéndola. La primera consistía en golpear el árbol para que cayeran los frutos, en la segunda se desprendían los frutos uno por uno del olivo. Era obviamente más costosa la segunda, pero daba aceitunas de mejor calidad.

En cuanto a las fechas de las actividades, lamentablemente ninguno de los documentos entrega datos. Fuentes notariales indican que los pagos de las deudas de las haciendas se efectuaban entre los meses de mayo y julio después de las cosechas $^{43}$. En consecuencia, se cosechaba la aceituna, al igual que en España, por la época de invierno.

\section{Elaboración de la aceituna}

Con respecto a la elaboración de la aceituna, tanto para su consumo como para la producción de aceite, tampoco hay una crónica específica del valle. Existe una descripción para Chile Central, de Gay, a comienzos del siglo XIX ${ }^{44}$. Hidalgo (1993:
37) sugiere que en Azapa se utilizó el segundo método durante el siglo XVIII. Documentos de carácter judicial $^{45}$, sin embargo, permiten reconstruir el proceso de elaboración de aceite en Azapa:

1. Se asoleaba la aceituna por dos días y medio.

2. Luego, para la molienda misma se cargaba la sal, la leña y la aceituna a los molinos. Los dos primeros eran los insumos de la elaboración y la aceituna era la materia prima.

3. El otro paso era moler la sal para la elaboración del aceite y aceitunas. Es decir, procesar uno de los insumos para que se pueda utilizar.

4. Se molía la aceituna en molinos tirados por caballos y se colocaba la pasta resultante en saquitos.

5. Por último, se prensaba la pasta en unas prensas accionadas por enormes tornillos que se apretaban con grandes vigas. Esta pasta, primero se prensaba sola, y luego mezclada con agua caliente para obtener la mayor cantidad posible de aceite.

En el documento sobre la administración de la hacienda de José Calderón se puede deducir, de la intervención de los peritos, que se producían en promedio 12 arrobas diarias libres de diezmos, primicias y maquila ${ }^{46}$. Con respecto a los medios de producción utilizados, un documento de 1775 sobre problemas de arriendo entrega una descripción de un molino, lo que permite establecer el nivel de la tecnología utilizada:

un molino para moler aseyte que se compone de piedra callana uisillo de prensa dos tinajas las ¿? ymfiernillo y la gloria [y en] su fondo que al parecer tendra setenta libras, un tablon $^{47}$.

También se conoce una descripción hecha a mediados del siglo XVII de otro molino:

$Y$ con su molino que muelen aseytuna y asen aseyte Y bodegas [sic.] Y [¿pul?]talla Vsillos y bigas de apretar aseyte $Y$ Uba para traer bino con todas sus paylas grandes $Y$ pequeñas $Y$ con las tinajas en que se Echa El aseyte que estan en la dicha vodega $Y$ su piedra Boladora mulas chacareras $Y$ con todo el demas apero (Hidalgo et al. 1990:94). 
Los documentos muestran que en Azapa existía, ya en el siglo XVII, una tecnología que en Chile Central sólo se conoció a mediados del siglo XIX. Es decir, funcionaba el molino de piedra, la glorieta, la prensa, el hornillo y las pailas para elaborar el aceite, además de las tinajas en las que se almacenaba el producto. Una parte de la producción del olivo, como indica Jofré Osorio, se destinaba a la aceituna "en caldo, machacada y pasada" 48 . Se supone que el sistema era similar al descrito por Gay:

Para prepararlas [las aceitunas], las pican con una espiga y se echan despues dentro de un vaso lleno de agua cambiándola dos veces al dia. Doce á quince dias bastan para quitarle todo su amargo y su alpachin ${ }^{49}$ [sic.], y se conservan despues con sal y comino seco (Gay 1973: 151).

Con respecto a la importancia relativa de los diversos tipos de trabajos que era necesario realizar, se puede indicar que para la mantención del árbol del olivo no se necesitaba mucha gente, a lo sumo un mayordomo y un regador, además del contrato de gente para ciertas tareas, como la poda y roza, la carga de guano en el período de crecimiento del fruto y limpiadores de las obras de regadío propias y colectivas. Sin embargo, al igual que el ají, la mayor parte de la mano de obra era utilizada en la época de preparación de la tierra y de cosechas, donde se requería mucha gente debido a las características del trabajo, la que según la Tabla 4 capturaba un $38 \%$ de toda la mano de obra utilizada en el año en uno a dos meses. Esta mano de obra de carácter estacional debió provenir de comunidades indígenas que debían vender su fuerza de trabajo a cambio de dinero para pagar las distintas obligaciones con la Corona y sus funcionarios (Hidalgo 1987: 224).

La diferencia con la producción del ají es que se necesitaba gente para el proceso de elaboración de la aceituna, tales como los cargadores de los frutos, de la sal, de la leña y del agua, además de los molineros, encargados del fuego y mayordomos de molinos, quienes debieron ser sujetos especializados. Al igual que con el ají, la producción de aceite en Azapa tenía condiciones específicas en el siglo XVIII, tanto por el mercado al que respondía como a la importancia de la cantidad que producía en el contexto de la oferta regional. El segmento del mercado al que llegaba este produc-
Tabla 4. Porcentaje de utilización de la mano de obra en las distintas actividades de la hacienda de Manuel Calderón.

Percentage of labor hours use for different activities at the Hacienda of Manuel Calderon.

\begin{tabular}{lc}
\hline Actividad & Porcentaje \\
\hline Labores culturales & 23 \\
Riego & 21 \\
Cosecha & 38 \\
Procesamiento & 18
\end{tabular}

Fuente: AJA, leg. 22, pza. 1, José Calderón. División de bienes en Azapa, Azapa 1781-1784, fjs. 25 r.-25 v.

to era la sociedad de origen hispano y la rica, aunque minoritaria, elite indígena. Éstas consumían aceite de oliva, principalmente como alimento de las capas altas de la sociedad. Y era el alimento sólo de las capas altas de la sociedad porque el olivo era un bien relativamente costoso que tenía un sustituto barato en la grasa de cerdo, muy utilizada por los demás sectores sociales con el mismo fin. Por esto Assadourian dice que este es un bien de consumo con neta discriminación social (Assadourian 1982: 163).

Según Tandeter y Wachtel (1983: Cuadro 3), hubo un proceso de aumento del precio del aceite a través del siglo XVIII, hasta finales de la década de 1780, para sufrir luego un proceso de estancamiento y leve disminución que dura hasta fines del período colonial. Es posible sostener que esto se debió al desarrollo del comercio interior con Europa y a un aumento de la producción de la plata, lo que trajo consigo la reactivación de la economía colonial. El segundo momento se explica por la saturación del mercado como el estancamiento de la producción de plata que hicieron decrecer la economía peruana (Assadourian 1982; Fisher 1977). Los efectos del crecimiento experimentado hasta la década de 1780 fueron aprovechados principalmente por los individuos de los estratos altos, lo que se tradujo en un mayor ingreso global para ese segmento. Esto trajo un mayor poder de compra de bienes normales o de lujo, con una alta elasticidad de ingresos como era el caso del aceite ${ }^{50}$.

En cuanto a la producción, aunque no se dispone de datos para fechas demasiado tempranas, sí existen para finales del siglo XVIII. Ponciano Jofré Osorio (ANA 1804) afirma que en Azapa se producían de 7.000 a 8.000 arrobas de aceite al año. 
Ureta y Peralta (1962 [1792]) sostiene que en el Corregimiento de Arica a fines del siglo de las luces se producían 40.000 arrobas $^{51}$. En consecuencia, podemos afirmar que en Azapa se producía del $17,5 \%$ al $20 \%$ de lo producido en el Corregimiento (Tabla 1). Ese porcentaje es mayor al ají a comienzos de siglo (13,33\%), cuando este producto era el principal del valle.

\section{Otros Productos: el Valle Dulce en Funcionamiento}

Aun cuando el olivo y el ají fueron en el siglo XVIII los productos principales del valle, no es menos cierto que también se cultivaban otras plantas, algunas de las cuales eran comerciales, pero ninguna tan importante en términos económicos como aquellas dos. Según Frezier, a comienzos del siglo XVIII en Arica (que se extendía para este ingeniero hasta la zona de Ocurica, adentrándose en el valle) se cultivaban "pequeñas praderas de alfalfa, cañas de azúcar, olivos i algodoneros mezclados con marismas cubiertas con totora", y desde San Miguel, sólo ají (Frezier 1902: 152). En tanto Ponciano Jofré Osorio (ANA 1804) sostiene que, hacia 1804, en Arica se obtenían

Cañas dulces, Mais, Alfalfa, Ajis Higueras, Platanos, algunos pocos Olibos, frutas como son Pacaes, Guayavas, Sandillas, Melones, Durasnos, Vbas; Legumbres detodas layas

y que en el valle se producían

Olibares, y algunos otros frutos como son Algodones, Aji, Mais, Papas, Sapallos, Cañas dulces Alfalfa y frutos de varias clases, y en particular Naranjas dulces, Limones, Cidras agrias, Chirimoyas, Platanos, Higos, Ubas, Guayavas, Sandillas Melones, Pacaes, y toda clase de Legumbres ${ }^{52}$.

Este carácter del valle de tener cultivos mixtos se reafirma también con los documentos que utilizamos en el análisis de la elaboración del aceite. En la hacienda de José Calderón hay información de que además de tener olivos se cultivaban alfalfa y aji ${ }^{53}$. El primero para uso doméstico y el segundo, aunque en un volumen muy pequeño, suponemos que se destinaba al mercado. En cuanto a la hacienda Arias, se afirmaba que los cultivos co- merciales eran el olivo y el algodón, pero el que dejaba mayores ingresos era el olivo. Además se explotaban con fines comerciales el maíz, la papa, el zapallo, las cebollas y los frijoles ${ }^{54}$. Y también se producía alfalfa para el consumo doméstico ${ }^{55}$.

\section{Conclusiones}

En este artículo se entregó una visión sistemática de dos procesos productivos, el del ají y el del aceite, en el valle de Azapa en el siglo XVIII. Se revisaron la geografía, los procesos productivos, las características y la evolución del comercio de estos productos y las relaciones de producción que se articulaban en torno a ellos. El marco geográfico (un valle dulce en una ubicación equidistante de los grandes centros urbanos coloniales) fue un estímulo para que se cultivaran, desde temprano, plantas para su comercialización. Junto con lo anterior, el que estos dos productos (ají y aceite) podían ser transportados a grandes distancias sin sufrir un proceso de descomposición orgánica, fue otro factor que favoreció el desarrollo de la producción de estos bienes.

La calidad de valle dulce se aprovechaba teniendo una gran variedad de cultivos. Sin embargo, los intereses económicos de aquellos que controlaban las rentas que se obtenían de la tierra (los grandes propietarios), en el siglo XVIII, ejercieron fuertes presiones para que se produjeran sólo los bienes de mayor valor comercial: ají y aceite, y en menor escala algodón. Esto fue en contra de los intereses de los administradores que vivían directamente vinculados a la tierra y que sufrían la inestabilidad económica provocada por estos monocultivos, ya que si bien eran productos con un crecimiento sistemático en sus precios, tendían a sufrir variaciones que hacían muy vulnerables a los que vivían sólo de su trabajo de la tierra. La prueba de que estuvieron en contra de estas presiones es que pese a las instrucciones de quienes controlaban el mercado, los administradores que se estudiaron en este artículo cultivaron más de un tipo de planta. Esto, tal vez, como un modo de dar estabilidad a su vida campesina.

El ají en particular, durante el transcurso del siglo XVIII, dejó de tener cierta importancia a escala regional, como lo demuestra la baja en la producción comparando las cifras entregadas por Frezier y Ureta y Peralta (1962 [1792]). Aunque sabemos la situación relativa que tendría el ají a 
escala regional en los comienzos del siglo XVIII, sí se conoce por Frezier que el ají era el producto principal de la zona a comienzos del siglo cuando realizó su visita a Arica. Y por Ureta y Peralta (1962 [1792]), que, aun cuando el ají seguía manteniendo el primer lugar en utilidades de la zona a fines del siglo XVIII, no es menos cierto que el algodón entregaba cifras parecidas y que el aceite tenía un rol importante en la economía regional. Más aún, este último producto, según los informes de Ponciano Jofré Osorio (ANA 1804), se fue posicionando en el transcurso del siglo como producto principal del valle (Tablas 1, 2 y 5).

Este fenómeno debió producirse por una modificación del mercado en términos cualitativos, es decir, en la composición de la demanda, lo que pudo traer consigo grandes cambios en la organización que se dio la sociedad agraria para poder pasar de la producción de un producto a otro, por el número de operarios que se necesitaban y los conocimientos técnicos que se precisaban para cultivar olivos y aceite ${ }^{56}$.

En cuanto a la importancia regional del valle, se puede sugerir que con la producción de aceite se logró tener una mayor preponderancia relativa que con el ají. Esta importancia tal vez estuvo dada por las ventajas que tenía con respecto a otros valles, tales como la condición de valle dulce y por la disponibilidad de tierras de cultivo no controladas por las comunidades indígenas (Hidalgo y Marsilli 1989), es decir, destinadas a una economía de subsistencia.

Tabla 5. Porcentaje de las ganancias totales que se obtenían por cada producto.

Percentage of total market income of each product.

\begin{tabular}{lcc}
\hline Frutos & Valores & \% de importancia en la zona \\
\hline Algodón & 160.000 & 26,55 \\
Ají de carga & 120.000 & 19,91 \\
Aceite & 120.000 & 19,91 \\
Vino & 112.500 & 18,67 \\
Ají regalado & 60.000 & 9,95 \\
Maíz & 30.000 & 4,97 \\
\hline Totales & 602.500 & 100,00 \\
\hline
\end{tabular}

Fuente: (Ureta y Peralta, 1965; fol. 132).
En el ámbito de la tecnología de la producción, se ha demostrado que las haciendas azapeñas se comportaban de un modo similar a lo sostenido por Wolf y Mintz (1975). De una parte, no existía una gran inversión en medios de producción, excepto para la elaboración final de los productos -especialmente en el caso del aceite-, utilizando la tecnología y las tradiciones culturales regionales para producir. Por ejemplo, la utilización de guano para intensificar la producción y el riego por caracoles. De otra, se ocupó relativamente poca mano de obra, con un incremento sólo en algunos períodos estratégicos estacionales, como la preparación del suelo, la siembra y la cosecha.

Finalmente, en lo que se refiere a las relaciones de producción, se lograron establecer los conflictos entre los distintos agentes con relación al uso de los recursos para la producción de bienes alternativos. Concretamente se mostró la contradicción entre los propietarios interesados en el monocultivo y los "agentes" o administradores directos de la tierra y el agua que preferían cultivar una variedad mayor de plantas para reducir los riesgos del mercado. Por otro lado, se estableció la existencia de diferentes oficios relacionados con la producción de estos bienes y el valor específico que tenían en el proceso productivo.

Agradecimientos: Este trabajo, en su primera versión, junto con la tesis que le dio origen (Henríquez 1998), formó parte del proyecto FONDECYT No 1980638 "Historia de los pueblos andinos de Arica, Tarapacá y Atacama: comunidades, cacicazgo y estado", cuyo investigador responsable fue Jorge Hidalgo Lehuedé. La versión actual contó con el soporte del proyecto FONDECYT N 1000089 "Historia de los Pueblos andinos de Arica, Tarapacá y Atacama: control político, memoria y resistencias étnicas, siglos XVI-XIX", a cargo de Jorge Hidalgo Lehuedé. No puedo dejar de reconocer las valiosas revisiones a los borradores y los aportes realizados por Luis Castro, Nelson Castro y Jorge Hidalgo, y las sugerencias editoriales y de contenido de los revisores de Chungara. Los errores, obviamente, son de mi entera responsabilidad. 


\section{Referencias Citadas}

AJA

1766 Fondo Judicial de Arica, Archivo Nacional de Chile. Legajo 60. pieza 4.

Álvarez, L.

1992 Lo dulce y lo salado: dos formas de percepción en los Andes, tiawanaku e inca. En Etnicidad, Economía y Simbolismo en los Andes. II Congreso Internacional de Etnohistoria, Coroico, pp. 147-161. HISBOL/IFEA/SBHSUR, La Paz.

ANA

Fondo Notarial de Arica, Archivo Nacional de Chile.

Assadourian, C.

1982 El Sistema de la Economía Colonial. Mercado Interno, Regiones y Espacio Económico. Instituto de Estudios Peruanos, Lima.

1979 La producción de la mercancía dinero en la formación del mercado interno colonial. En Ensayos sobre el Desarrollo Económico de México y América Latina, 1500-1975, editado por E. Florescano, pp. 223-292. Fondo de Cultura Económica, México.

Burga, M

1976 De la Hacienda Colonial a la Hacienda Capitalista. Instituto de Estudios Peruanos, Lima.

Burzio, $\mathrm{H}$

1958 Diccionario de la Moneda Americana, vol. 1. Fondo Histórico y Bibliográfico José Toribio Medina, Santiago.

Castro, L.

1999 Cuando el Susurro del Agua se Acalló en el Desierto: la Expropiación de las Aguas del Valle de Quisma, el Abastecimiento Fiscal a Iquique y el Debate sobre el Problema del Desarrollo Económico Regional. Tarapacá 1912-1937. Tesis de Magíster en Historia, Universidad de Santiago de Chile, Santiago.

CORFO

1950 Geografía Económica de Chile. Tomo 1. Editorial Universitaria, Santiago.

Chonchol, J.

1996 Sistemas Agrarios en América Latina. De la Etapa Prehispánica a la Modernización Conservadora. Fondo de Cultura Económica, Santiago.

Errázuriz, A., J. González, M. González, M. Henríquez, P. Cereceda y R. Rioseco

1987 Manual de Geografía de Chile. Editorial Andrés Be1lo, Santiago.

Figueroa, C.

2001 Riego y Sociedad: Pica Siglo XVIII. Tesis de Licenciatura en Historia, Universidad de Valparaíso, Valparaíso.

Fisher, J.

1977 Minas y Mineros en el Perú Colonial, 1776-1824. Instituto de Estudios Peruanos, Lima.

Frezier, M.

1902 Relación del Viaje por el Mar del Sur y las Costas de Chile y el Perú Durante los años de 1712, 1713 y 1714. Imprenta Mejía, Santiago.

Garavaglia, J.

1983 Mercado Interno y Economía Colonial. Editorial Grijalbo, México.

Gay, C.

1973 Agricultura Chilena. 2 Vols., ICIRA, Santiago.
Glave, L.

1983 Trajines: Un capítulo en la formación del Mercado interno colonial. Revista Andina, año 1, tomo 1: 9-76

Gómez de Fuencarral, J.

1883 Cultivo del Olivo. Gaspar Editores, Madrid.

Henríquez, F.

1998 Hacienda y Economía Colonial: Azapa en el Siglo XVIII. Tesis de Licenciatura en Historia. Universidad de Valparaíso, Valparaíso.

Hernández, M.

2000 Viento del Pueblo. Editorial Lumen, Barcelona.

Hidalgo, J.

1987 Tierras, exacciones fiscales y mercado en las sociedades andinas de Arica, Tarapacá y Atacama, 1750-1790. En La Participación Indígena en los Mercados Surandinos, editado por O. Harris, B. Larson y E. Tandeter, pp. 193235. Ed. CERES, La Paz.

1993 Algunas notas para el estudio del olivo en Arica (Chile). Idesia 12:31-50.

Hidalgo, J. y M. Marsilli

1989 La cabecera del valle de Azapa en el siglo XVII. El sector de Chirispaya en la expansión de las haciendas tempranas. Manuscrito en posesión de los autores.

Hidalgo, J., M. Marsilli, C. Santoro y R. Correa

1990 Compraventa de una hacienda en el valle de Azapa por Gaspar de Oviedo, 1661. Documento inédito del Archivo de la Nación. Lima Perú. Diálogo Andino 9: 84-106.

Instituto Geográfico Militar

1985 Geografía de Chile. Tomo I, Primera región de Tarapacá. Instituto Geográfico Militar, Santiago.

Keller, C.

1946 El Departamento de Arica. Ministerio de Economía y Comercio, Secretaría General del Censo Económico. Santiago.

Mörner, M. y F. Martínez

1980 Medidas como precios y como instrumentos para la explotación. Un expediente cuzqueño del siglo XVIII. Allpanchis 15:133-150.

Murra, J.

1975 Formaciones Económicas y Políticas del Mundo Andino. Instituto de Estudios Peruanos, Lima.

Platt, T.

1975 Experiencia y experimentación: los asentamientos andinos en las cabeceras del valle de Azapa. Chungara 5: 33-60.

Rivera, M.

1983 Patrones Prehispánicos y Contemporáneos del uso de la tierra en el Valle de Azapa, Norte de Chile. Diálogo Andino 2:11-25.

Romano, R.

1992 Consideraciones. Instituto Italiano de CulturaFONCIENCIA. Lima.

Rostworowski de Diez Canseco, M.

1981 Recursos Naturales y Pesca, Siglos XVI y XVII. Instituto de Estudios Peruanos, Lima.

Takahashi, H.

1986 Vino en Arequipa, migración y trabajo en el sur andino: siglo XVI. En Etnografía e Historia del Mundo Andino. 
Continuidad y Cambio, editado por Shozo Masuda, pp. 109138. Universidad de Tokio, Tokio.

Tandeter, E.

1992 Coacción y Mercado. La Minería de la Plata en el Potosí Colonial, 1692-1826. Editorial Sudamericana, Buenos Aires.

1995 Población y economía en los Andes (siglo XVIII). Revista Andina año 13, 1:7-22.

Tandeter, E. y N. Wachtel

1983 Precios y Producción Agraria. Potosí y Charcas en el Siglo XVIII. Ediciones CEDES, Buenos Aires.
Ureta y Peralta, P.

1962 [1792] Descripción de la Ciudad de Arica y su vasta jurisdicción correspondiente a la Intendencia de Arequipa en el Perú. Mercurio Peruano Lima, Nos 188, 189, 190, 191, Vol. VI: f. 113 r. - 145 r.

Wolf, E. y S. Mintz

1975 Haciendas y plantaciones en Mesoamérica y las Antillas. En Haciendas, Latifundios y Plantaciones en América Latina, editado por E. Florescano, pp. 493-531. CLACSO-Siglo XXI, México.

Wrigley, E.

1994 Historia y Población. Introducción a la Demografía Histórica. Editorial Crítica, Barcelona.

\section{Notas}

1 Véase Henríquez (1998), específicamente los capítulos V y VII, relacionados con los propietarios y las relaciones de producción, respectivamente.

2 Para el caso de la minería hay abundantes y excelentes trabajos. Tal vez los más destacados en la zona andina son los de Assadourian sobre la huayra y el amalgamamiento en Potosí a comienzos del período colonial (Assadourian 1979) y de Tandeter sobre la minería también en Potosí durante fines del período colonial (Tandeter 1992).

3 Archivo Nacional de Chile, Fondo Notarial de Arica (de ahora en adelante ANA) vol. 4, Informe de Ponciano Jofré Osorio en Arica , Arica 1804, fj.41 v.

4 En la actualidad el valle no se extiende hasta Las Chimbas y ha extendido varios kilómetros sus hectáreas cultivables hacia el oriente, debido principalmente de las aguas de que se pudo disponer por el canal "Lauca". Un estudio clásico respecto al impacto en la sociedad contemporánea de este proceso se encuentra en el artículo de Platt (1975) respecto a la utilización andina de las tierras que quedaron disponibles.

5 Según Frezier, en la primera legua del valle, antes de llegar a San Miguel, se cultivaban alfalfa, caña de azúcar olivos y algodoneros. (Frezier 1902:152). Esto sería una prueba de lo diversificada de la producción, aunque se destaca la ausencia de viñas, tan importantes para la economía local en el siglo XVII.

6 " $[. .$.$] Los caracoles consisten en una S, de ángulos rectos,$ cuyas vueltas pueden continuarse indefinidamente. El agua corre despacio por estas vueltas y las plantas se colocan justo al borde del agua para que no se mojen demasiado. El caracol no necesita una profundidad de suelo muy grande, y en los lugares donde el ripio brota en la superficie de la tierra, los caracoles se construyen siguiendo en lo posible los huecos entre las piedras. Con leves variaciones en la pendiente del caracol se puede controlar con precisión la cantidad de agua que llega a la planta."... (Platt 1975: 4849)

7 Ver notas 38 y 39.

8 Ver lo dicho a este respecto en el apartado anterior.

9 Archivo Nacional de Chile, Fondo Judicial de Arica (de ahora en adelante AJA), leg. 1, pza. 6, Juan Domínguez Aguilar contra Baltazar Pérez de los Ríos. Cobro de pesos en Azapa, Azapa 1735-1737, fj. 11 r.

10 Ibid. fj. 13 r.
11 Ibid. fj. 12 r.

12 Ibid. fj. $21 \mathrm{r}$.

13 En AJA leg. 1 p. 18 "Manuel Albarracín contra Bentura Iglesias, cobro de pesos en Azapa" (1794-1810), hay sólo una indicación de transporte de productos a Arica (f. 57 r.), así es que presumimos que lo demás era vendido en la misma hacienda. Para mayores antecedentes sobre la vinculación de los hacendados véase Henríquez (1998), específicamente el capítulo quinto.

14 En realidad afirma que eran 80.000 escudos, pero creemos que esto se debe a un error, ya que teniendo presente que en el siglo XVIII un escudo de plata salía entre 12 y 16 pesos (Burzio 1958), se obtiene como resultado una producción de 960.000 ó 1.280.000 pesos, cifras superiores al total regional.

15 AJA, leg. 1, pza. 6, Juan Domínguez Aguilar contra Baltazar Pérez de los Ríos. Cobro de pesos en Azapa, Azapa 1735-1737, fj. 21 r.

16 AJA, leg. 106, pza. 6, Pedro Martín Carrasco contra María Jiménez y Arias. Sobre hacienda en Azapa, Azapa 17601761, fj. $118 \mathrm{r}$.

17 Esto es válido si se acepta, sólo para tener un punto de comparación, que una arroba equivale a 11,5 kg (Mörner y Martínez 1980: 135). Aunque lo más probable es que no haya correspondido exactamente a ese valor ya que las medidas hasta épocas recientes eran muy relativas, y respondían a factores tanto geográficos como económicos (Mörner y Martínez 1980: 133 y 137).

18 ANA, vol. 4, Informe de Ponciano Jofré Osorio en Arica, Arica 1804, fjs. 41 r.-42 v.

19 (Ureta y Peralta 1962: fol. 132). Aún cuando las cifras dadas por este autor están redondeadas, nos sirven para darnos una idea general de lo que pasaba en la región.

20 La Corona española trató sistemáticamente de frenar el proceso de especialización de las haciendas costeras peruanas en viñedos y principalmente en olivares con el objeto de eliminar la competencia en las colonias de los productores peninsulares de esos productos (Chonchol 1996: 72).

21 Ver el apartado referido al ají.

22 Para información sobre el modo en que se introdujeron en América ver Hidalgo (1993).

23 AJA, leg. 1, pza. 18, Manuel Albarracín contra Bentura Iglesias, cobro de pesos en Azapa, Azapa 1794-1810, fjs. 53 r. $-58 \mathrm{v}$. 
24 AJA, leg. 1, pza. 18, Manuel Albarracín contra Bentura Iglesias, cobro de pesos en Azapa, Azapa 1794-1810, fjs. 53 r. - 58 v.; y AJA, leg. 22, pza. 1, José Calderón. División de bienes en Azapa, Azapa 1781-1784, fjs. 25 r. - 25 v.

25 Con respecto al agua y su utilización como recurso, las ideas expresadas en este artículo se encuentran fundamentadas en trabajos sobre la zona de Tarapacá. Específicamente el de Figueroa (2001) sobre el oasis de Pica y el de Castro (1999) sobre el valle de Quisma.

26 AJA, leg. 22, pza. 1, José Calderón. División de bienes en Azapa, Azapa 1781-1784, fj. 29 r.

27 Se debe tener en cuenta que los peritos son, al igual que los que demandan, grandes hacendados que tienen intereses en las rentas de la tierra sin depender directamente de ella.

28 AJA, leg. 1, pza. 18, Manuel Albarracín contra Bentura Iglesias, cobro de pesos en Azapa, Azapa 1794-1810, fj. $48 \mathrm{r}$.

29 Ibid. fj. 69 r.

30 Por ejemplo, para un contexto distinto, en términos del producto, como es la producción de trigo en Inglaterra en épocas preindustriales, Wrigley sostiene que "[...] La zona de cultivo mixto es más sana que la zona que depende principalmente de los caprichos de la cosecha de trigo"... (Wrigley 1994: 99). Teniendo en cuenta esto, se puede sostener que en general donde se genera el monocultivo en épocas preindustriales se depende mucho de que existan las condiciones climáticas adecuadas sólo para un tipo de planta. En cambio, en los cultivos mixtos, las condiciones que perjudican a un tipo de cultivo pueden beneficiar a otro y así compensar las pérdidas

31 AJA, leg. 1, pza. 18, Manuel Albarracín contra Bentura Iglesias, cobro de pesos en Azapa, Azapa 1794-1810, fj. $57 \mathrm{r}$.

32 Ibid fj. $56 \mathrm{r}$.

33 Ibid fj. $53 \mathrm{v}$.

34 Ibid fj. $55 \mathrm{r}$.

35 AJA, leg. 22, pza. 1, José Calderón. División de bienes en Azapa, Azapa 1781-1784, fj. 25 r.

36 El camayo, dentro de este ámbito es el individuo que estaba a cargo de la hacienda, es decir, el que la administraba en reemplazo de los dueños. También en la documentación son denominados mayordomos.

37 Específicamente, el que tenía a cargo la hacienda, en la rendición de cuentas justificó 100 pesos de gastos "Por dos años que he mantenido camayo". Este salario es menor de lo que recibiría un peón que ganara seis reales durante todo el año (es lo que se acostumbraba pagarle a los peones en Azapa). Y el carácter de su labor se perfila también en la explicación que hace Manuel Calderón de su contratación ante los reparos de su madrastra Joaquina Zavala: “[...] El motivo que hai para mantener halli camaio es forsoso por que no pasando en la hazienda devo dejar una persona que la cuide evitando los daños que la imbadan quedando desierta en la soledad"... AJA, leg. 22, pza. 1, José Calderón. División de bienes en Azapa, Azapa 1781-1784, fjs. 25 r.; $36 \mathrm{v}$

38 AJA, leg. 1, pza. 18, Manuel Albarracín contra Bentura Iglesias, cobro de pesos en Azapa, Azapa 1794-1810, fjs. $53 \mathrm{r} .-58 \mathrm{v}$.

39 Ibid, fjs. 53 r. -58 v.
40 AJA, leg. 22, pza. 1, José Calderón. División de bienes en Azapa, Azapa 1781-1784, fjs. 25 r. - 25 v.

41 AJA, leg. 1, pza. 18, Manuel Albarracín contra Bentura Iglesias, cobro de pesos en Azapa, Azapa 1794-1810, fjs. $53 \mathrm{r} .-58 \mathrm{v}$.

42 Ibid f. $53 \mathrm{v}$.

43 Véase ANA vol. 1 f. 74 v. - 78 r. "Venta Real Domingo López de Santana a nombre de Sebastián Núñez a Feliciano Osorio en Azapa" (1732); ANA vol. 1 f. 80 v. - 81 r. "Obligación de Francisco Javier Portocarrero al Doctor Alfonso Diez en Azapa" (1731); ANA vol. 20 f. 13 v. - 14 v. "Arrendamiento de una hacienda, Gerónimo Salgado Araujo a Cayetano de Oviedo en Azapa" (1731); ANA vol. 20 f. 18 v. - 19 v. "Arrendamiento de una hacienda, Juan Jiménez de Valverde a Narciso Cañipa en Azapa” (1731); AJA leg. 154 p. 7 "Rafael Rodríguez contra Agustín Ferrer. Sobre arrendamiento de hacienda en Arica" (1775).

44 Gay sostiene que en la zona central de Chile se habrían utilizado dos métodos de elaboración de aceite, uno muy poco eficiente, que producía aceite de mala calidad, y otro más eficaz, que sería una copia del utilizado en Europa en esos momentos. Con respecto al primer método, sostiene lo siguiente:

despues de guardarlas [las aceitunas] en monton par [sic.] cinco á seis dias y á veces hasta un mes y presadas con piedras se ponen sobre un plano inclinado y con agua que ha de ser muy caliente, al punto que causa con frecuencia un dolor irresistible al operario. El líquido va á reunirse en una batea ú otro vaso y despues de enfriarlo se saca por medio de una cuchara el aceite que sobrenada en el agua y que se clarifica al fuego. Se bota el bagazo como inútil ó se emplea para alimentar á los hornos, conteniendo todavía mucho aceite por el vicioso con que se ha operado (Gay 1973: 151).

En resumen, era una técnica que se basaba en la utilización de la fuerza humana directamente, la cual por ser insuficiente no permitiría aprovechar el fruto al máximo.

Haciendas modernas cercanas a Santiago hicieron uso de la siguiente técnica de producción de aceite:

las aceitunas, cinco dias despues de cosechadas, se muelen en un molino movido por un caballo. Las materias reducidas en pasta se echan en varios saquitos para apresarla por medio de una fuerte prensa, primeramente con moderación para tener aceite de primera calidad, y despues mezclada con agua caliente se le da otra compresion mucho mas fuerte para conseguir todo el aceite contenido y que se vende por segunda y tercera calidad (Gay 1973: 151).

La eficiencia de esta técnica de producción, que combinaba el uso de energía animal para mover la piedra del molino con la utilización de prensas para obtener el producto final, es mayor que la anterior, pues multiplicaba la fuerza empleada por los hombres, logrando un mejor aprovechamiento de la materia prima y una mayor producción de aceite.

45 Estos pasos están tomados de las rendiciones de cuentas que aparecen en AJA, leg. 1, pza. 18, Manuel Albarracín contra Bentura Iglesias, cobro de pesos en Azapa, Azapa 1794-1810, fjs. 53 r. - 58 v.; AJA, leg. 22, pza. 1, José Calderón. División de bienes en Azapa, Azapa 1781-1784, fjs. $25 \mathrm{r} .-25 \mathrm{v}$. 
46 Esto lo deducimos de la producción dada en la rendición de cuentas, dividida por el número de días que dijeron los expertos que se demoraban en producir esa cantidad. En AJA, leg. 22, pza. 1, José Calderón. División de bienes en Azapa, Azapa 1781-1784. Los diezmos, primicias y maquilas son cantidades de producto que se deben entregar, las primeras dos a la Iglesia, y la tercera al dueño del molino.

47 AJA, leg. 154, pza. 7, Rafael Rodríguez contra Agustín Ferrer. Sobre arrendamiento de hacienda en Arica, Arica 1775 , fj. 5 r.

48 ANA, vol. 4, Informe de Ponciano Jofre Osorio en Arica, Arica 1804, fjs. 41 r. - 42 v. En AJA, leg. 1, pza. 18, " $M a$ nuel Albarracín contra Bentura Iglesias, cobro de pesos en Azapa, Azapa 1794-1810, se informa que una parte de la producción de oliva se vende como aceituna.

49 Alpechín es el líquido oscuro y fétido que sale de las aceitunas apiladas.
50 Se entiende por bienes normales aquellos que el consumidor compra en mayores cantidades cuando su nivel de ingresos se incrementa, efectuándolo en la misma proporción o en una proporción inferior a éstos. Y los bienes de lujo son aquellos que el consumidor compra en mayores cantidades cuando su nivel de ingresos se incrementa, realizándolo en una proporción mayor a éstos.

51 (Ureta y Peralta 1962: fol. 132).

52 ANA, vol. 4, Informe de Ponciano Jofre Osorio en Arica, Arica 1804, fjs. 41 r. - 42 v.

53 AJA leg. 22 p. 1, José Calderón. División de bienes en Azapa", Azapa, 1781-1784, fjs. 29 r., 31 v.

54 leg. 1, pza. 18, Manuel Albarracín contra Bentura Iglesias, cobro de pesos en Azapa, Azapa 1794-1810, fj. 58 r.

55 Ibi, fj. 55 v., por ejemplo.

56 Una aproximación al problema de la mano de obra para el valle se puede encontrar en (Henríquez 1998), en especial el séptimo capítulo, dedicado a las relaciones de producción en el valle. 